\title{
A Case of Pulsatile Tinnitus from the Atherosclerosis and Atheroma in Superior Labial Artery and Facial Artery
}

\author{
Jung Min Kim, Choon Dong Kim and Seung Woo Kim \\ Department of Otolaryngology-Head and Neck Surgery, VHS Medical Center, Seoul, Korea
}

\author{
Received May 3, 2012 \\ Revised September 30, 2012 \\ Accepted December 2, 2012
}

\section{Address for correspondence \\ Seung Woo Kim, MD \\ Department of Otolaryngology- \\ Head and Neck Surgery, \\ VHS Medical Center, \\ 53 Jinhwangdo-ro 61-gil, \\ Gangdong-gu, Seoul 134-791, \\ Korea \\ Tel $+82-2-2225-1384$ \\ Fax +82-2-2225-1385 \\ E-mail drdrum@dreamwiz.com}

Tinnitus is one of the most common symptoms in an audiologic field. It can be classified as either as subjective or objective; former referring to the sensation heard by both patient and examiner. Pulsatile tinnitus is perceived as sounds that vary in frequency, intensity and duration. The cause of pulsatile tinnitus include high jugular bulb, benign intracranial hypertension, glomus tumors, carotid artery stenosis, vascular lesions of the temporal bone, arteriovenous malformation, aneurysms, and Arnold-Chiari malformation. Vascular tinnitus is most common (7.6\%). Recently, the authors experienced one case of the pulsatile tinnitus caused by atherosclerosis and atheroma in superior labial artery \& facial artery. After surgery, the symptom had disappeared. We report a unique case regarding the objective tinnitus with the literature review.

Korean J Audiol 2012;16:156-158

\section{Introduction}

Tinnitus is the most common symptom in the field of otolaryngology. It refers to abnormal hearing of sound heard during metabolism and not from an external sound source. The symptom is commonly categorized as vibratory and non-vibratory tinnitus. The former is divided into objective and subjective tinnitus. The most common cause of vibratory tinnitus is intracranial vascular disease, such as aneurysm and arteriovenous malformation. ${ }^{1)}$

The authors performed excisional biopsy on pulsatile lip masses of 46 year old male. Pathology confirmed vasculature with atherosclerosis and atheroma. The tinnitus was cured with removal of the mass. We report a unique case regarding the objective tinnitus with review of the literature.

\section{Case Report}

A 46 year old male patient experienced vibratory tinnitus on the right side for 10 years. He had pulsatile mass on the right side of his lip and did not undergo any treatment. Since 1 month before diagnosis, the level of tinnitus increased along with the size of the mass. Past medical history shows both lower limbs amputation 20 years ago and hypertension. Upon physical examination, there were no symptoms of tenderness, but 2 pulsatile masses were found on the right upper and right lateral side lip. The size of the former mass was $1 \times 1 \mathrm{~cm}$ and the latter was $1.5 \times 1.0 \mathrm{~cm}$ (Fig. 1). There were no other special abnormalities in the oral cavity, oropharynx and the other head and neck area. The tinnitus decreased when pressure was applied to the left tympanic membrane area and increased when leaning forward. The tympanic membrane was in sound condition and hearing tests revealed both ears to be normal. Similar observations were made during two times of tinnitogram. The frequency was $2 \mathrm{kHz}$, and loudness was between 60 and 70 decibels and there were no residual inhibitions. Arteriovenous malformation from the right facial artery was found resulting from computed tomography of the facial bone (Fig. 2). CT with angiography was not conducted due to hypotension and tachycardia after the injection of the contrast media. It was diagnosed as resulting from an abnormality in vessel and excisional biopsy was conducted through oral access after general anesthesia (Fig. 3). Operation revealed the superior labial artery and facial artery after incising the oral mucosa in the mass and the area showing evidence of vascular malformation was ligated. The area was irrigated and operation was 
concluded. Patient was allowed to return home 3 days after the operation. Pathology diagnosed the condition as atherosclerosis (Fig. 4). The patient did not complain of any tinnitus symptoms after the operation and shows no sign of recurrence up

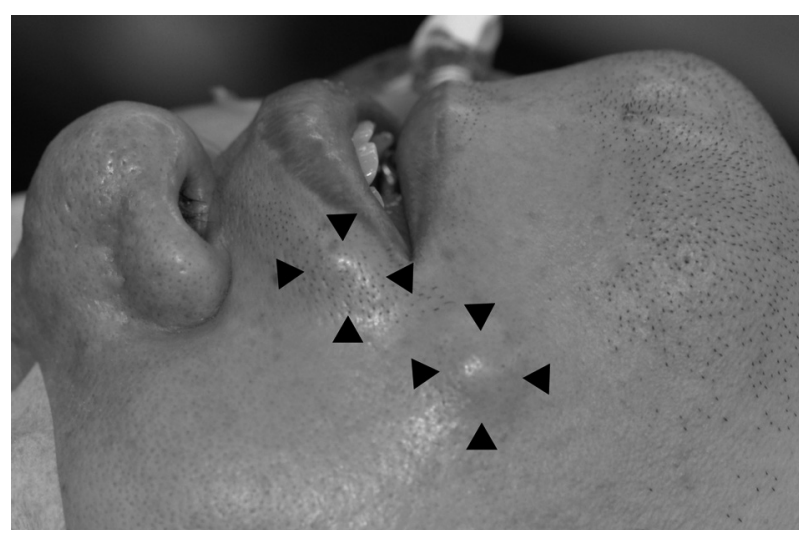

Fig. 1. It shows two pulsatile soft mass in the right side of the mouth angle and superior to the upper lip. until now, 1 year after the operation.

\section{Discussion}

Tinnitus can be classified as objective or subjective tinnitus, depending on whether the examiner can hear the sound that the patient is complaining about. From a radiologic point of view, it can be categorized into pulsatile tinnitus and non-pulsatile tinnitus. The common causes of objective tinnitus are abnormalities in the vessel. Non-vascular causes include the abnormal patency of the eustachian tube, temporomandibular joint disorder, arteriovenous anastomosis, myoclonus of the oropharyngeal, stapedius, and tensor tympani muscles. ${ }^{2)}$ Depending on the type of condition, pulsatile tinnitus shows varying levels of strength, duration and the size of the tinnitus, all of which are characteristically in line with the regular rhythm of the patient's heart beat. Sometimes true pulsatile and pseudo-pulsatile tinnitus is categorized by whether the tinnitus is

Fig. 2. The computed tomography (neck, enhanced) shows heterogenous enhancement in the right upper lip $(A$, white arrow) and right buccal area $(B$, white arrow head).
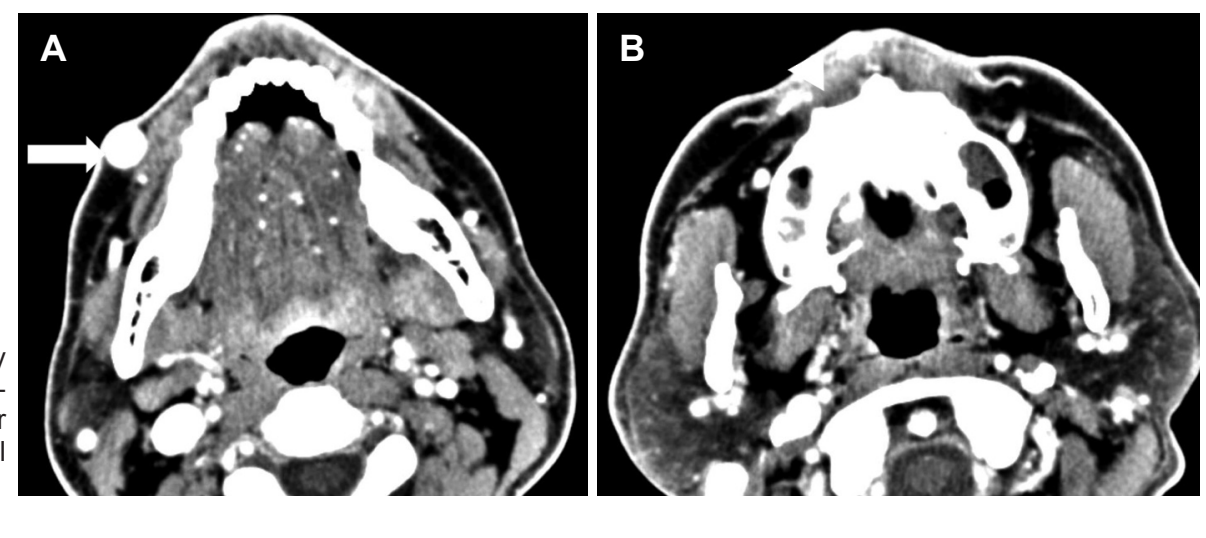

Fig. 3. Intraoperative findings. A: The mass is located on the right upper lip. It was slightly adhered but was dissected without much difficulty. B: The mass is located on the right buccal area. It was same as ' $A$ ', but smaller.
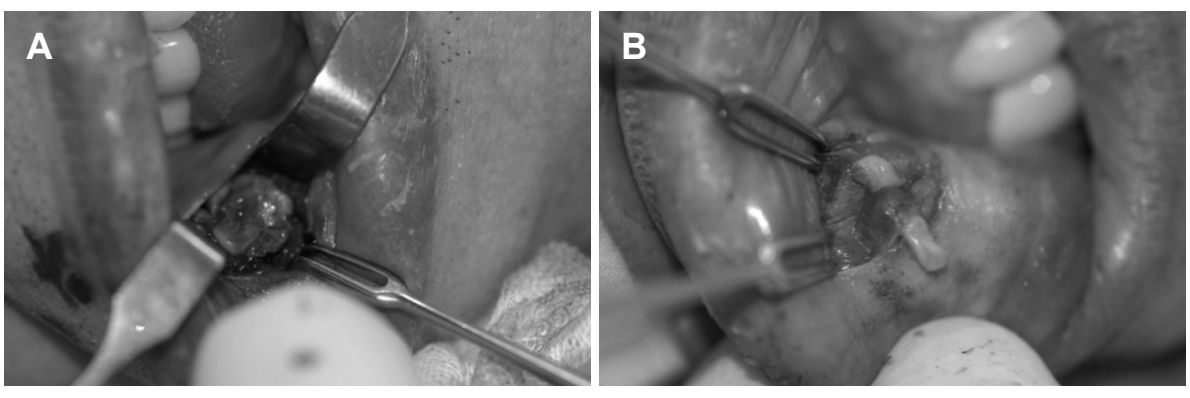

Fig. 4. These photographs show gross and microscopic findings. A: The gross specimen shows two pieces of irregular-shaped, pinkish gray and soft tissue, measuring $1.6 \times 1.0 \times 0.3$ $\mathrm{cm}$ and $1.5 \times 0.8 \times 0.3 \mathrm{~cm}$. B: Vasculature with atherosclerosis and atheroma (H\&E, $\times 100)$.
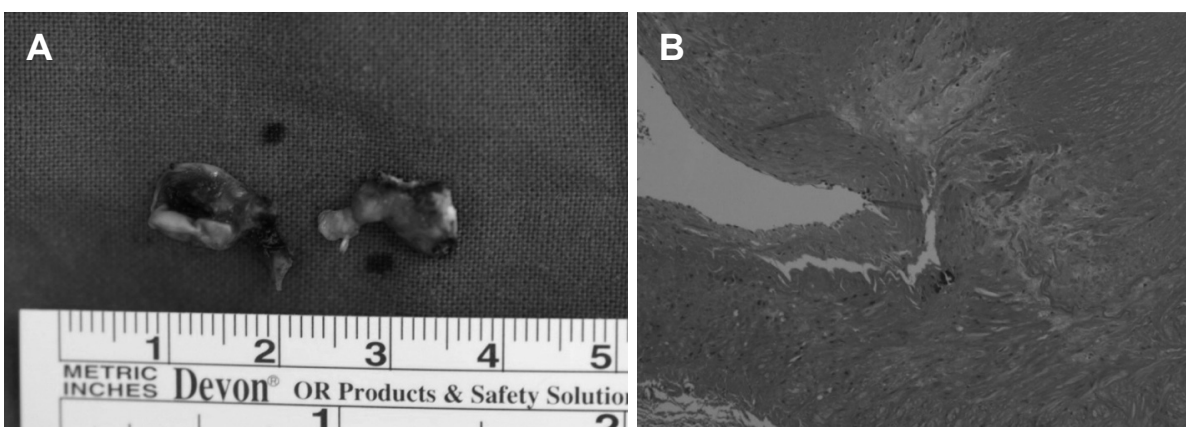
in line with the rhythmic beating of the heart. True pulsatile tinnitus may be a sign of vascular disease. Patients diagnosed with objective tinnitus mostly show vascular disease representing a $7.6 \%$ of all tinnitus patients. ${ }^{3)}$ Finding the cause of the tinnitus raises the possibility of cure, and tinnitus can sometimes be the tell-tale sign of a more serious condition. Thus, finding the cause of the tinnitus is of utmost importance.

Clinical symptoms differ depending on the location of the lesion and the structure of the anatomy. If the lesion is close to the skull base or scalp vessel, symptoms such as pulsatile mass, palpable thrill or murmur may show and pulsatile tinnitus. $^{4,5)}$

The possible venous cause for pulsatile tinnitus is benign intracranial hypertension and intracranial venous malformation. Benign intracranial hypertension usually shows in young obese females, and symptoms include pulsatile tinnitus, headaches, blurred vision among other non-characteristic symptoms. Otologic symptoms, such as earfullness, low frequency hearing difficulty and dizziness may also follow. A total of $80 \%$ of pulsatile tinnitus shows unilateral. Symptoms worsen during situations where the cranial blood pressure increases, such as coughing and leaning forwards and thus, are at its peak during the morning. Pulsatile tinnitus arising from venous malformation can be due to sigmoid sinus diverticulum, jugular bulb diverticulum, dehiscent high jugular bulb, dehiscent sigmoid sinus and abnormal emissary vein. However, the abnormalities in the vein do not always result in tinnitus.

Patients with pulsatile tinnitus must be examined thoroughly, including neurological and radiological examination, along with physical examination and past history. In cases with vessel malformation, angiography must be utilized to find the exact location of the lesion. A common neoplasm of pulsatile tinnitus is glomus tumor. Other neoplasms include middle ear adenoma, hemangioma, hemangiopericytoma, plasmacytoma, giant cell tumor, neuroendocrine carcinoma, and etc. In this example, the location of the lesion was relatively easier to access, and due to the abnormal reaction, the CT-angiography was not conducted.

For a radiological examination of pulsatile tinnitus, enhanced temporal bone-HRCT, enhanced brain CT, CT angiogra- phy, MR angiography/venography, four-vessel angiography and the contrast enhancement are used to observe the head and neck vessel in detail. Which examination method should be used differs depending on the examiner. This is because the diagnostic sensitivity and the specificity of each method differ, and as there is no specified standard in determining the level of the finding to be considered abnormal. However, to propose some rules to the method of examination, firstly, a method utilizing the contrast enhancement must be used, and secondly, a method checking the abnormalities in angiography in the cervical and head, not just in the temporal bone, must be used. Thirdly, if there are past history in the head trauma and there is a possibility of an arterial malformation and no abnormalities are found upon initial exam, four-vessel angiography must be considered. ${ }^{6}$

External and internal access was considered before the surgery, however due to the young age of the patient and for cosmetic reasons, the area was accessed through the mouth. The mass was successfully removed and the tinnitus completely disappeared. It is considered that this instance of tinnitus arising from the facial vessel is very abnormal. The authors successfully cured tinnitus after removal of arteriovenous malformation near the lips and have compiled the findings in this thesis.

\section{REFERENCES}

1) Rich NM, Hobson RW 2nd, Collins GJ Jr. Traumatic arteriovenous fistulas and false aneurysms: a review of 558 lesions. Surgery 1975; 78:817-28.

2) Scamoni C, Dario A, Basile L. The association of cavernous and venous angioma. Case report and review of the literature. Br J Neurosurg 1997;11:346-9.

3) Yoo HJ, Park SN, Kim DK, Park KH, Kim MJ, Kim JE, et al. Incidence and clinical characteristics of patients with tinnitus according to diagnostic classification. Korean J Otorhinolaryngol-Head Neck Surg 2011;54:392-8.

4) Presutti L, Laudadio P. Jugular bulb diverticula. ORL J Otorhinolaryngol Relat Spec 1991;53:57-60.

5) Del Curling O Jr, Kelly DL Jr, Elster AD, Craven TE. An analysis of the natural history of cavernous angiomas. J Neurosurg 1991;75:702-8.

6) The Korean Otologic Society. Imaging for tinnitus. In: Lee HJ, editors: Choi CH. Current Opinion on tinnitus. 1st ed. The Korean Otologic Society;2010. p.97-8. 